\title{
Comparative Efficacy of Doxycycline vs. Azithromycin in Pediatric Scrub Typhus
}

\author{
Thirunavukkarasu Arun Babu ${ }^{1}$ (D) $\cdot$ Dinesh Kumar Narayanasamy ${ }^{2} \cdot$ Prakash Mathiyalagen $^{3}$
}

Received: 11 May 2020 / Accepted: 4 August 2020 / Published online: 14 August 2020

(C) Dr. K C Chaudhuri Foundation 2020

To the Editor: Scrub Typhus (ST) is a re-emerging, acute, febrile infectious disease caused by gram-negative bacterium, Orientia tsutsugamushi [1]. Doxycycline is the drug of choice for Pediatric ST while Azithromycin is considered as an effective alternative $[2,3]$. However there are no clear evidence based guidelines to choose between these two drugs [1, 4]. This study was done to compare the efficacy of both these drugs in Pediatric ST.

All children $\leq 12$ y with fever $\geq 7 \mathrm{~d}$ and positive IgM ELISA for ST (InBios International, Inc., USA; Optical Density OD $>0.5$ was considered positive) were included. These children received either Azithromycin $(10 \mathrm{mg} / \mathrm{kg} / \mathrm{d}$ PO once a day for 7 d) or Doxycycline (For children <40 kg: $2.2 \mathrm{mg} / \mathrm{kg}$ twice daily and > $40 \mathrm{~kg}$ : $100 \mathrm{mg}$ twice daily for $7 \mathrm{~d}$ ) [1]. Children treated with antibiotics prior to diagnosis and those with noncompliance were excluded. Fever Clearance Time (FCT- time between first dose of antibiotic to the time when the temperature falls $\leq 37.5^{\circ} \mathrm{C}$ and remains so for at least $24 \mathrm{~h}$ ), duration of hospitalization and other clinical characteristics of cases in both groups were compared.

Out of total $510 \mathrm{ST}$ cases, 267 (52.3\%) received azithromycin and $243(47.6 \%)$ cases got doxycycline. FCT was significantly lower in Doxycycline group (42 $\pm 24 \mathrm{~h}$ ) when compared to Azithromycin $(48 \pm 23 \mathrm{~h})(p=0.001)$. Persistence of fever for $>48 \mathrm{~h}$ after starting antibiotic was significantly less in Doxycycline group $(p=0.001)$. There was no difference in duration of hospital stay and outcome

Thirunavukkarasu Arun Babu

babuarun@yahoo.com

1 Department of Pediatrics, All India Institute of Medical Sciences (AIIMS), Mangalagiri, Andhra Pradesh 522 503, India

2 Department of Pediatrics, JIPMER, Karaikal, Pondicherry, India

3 Department of Community Medicine, Indira Gandhi Medical College and Research Institute, Pondicherry, India between these two groups. Mean FCT in Doxycycline group was significantly lower when age $>5$ y $(p=0.003)$, second week of fever $(p=0.003)$, presence of abdominal symptoms $(p=0.003)$, facial puffiness $(p=0.003)$, generalised lymphadenopathy $(p=0.003)$, hepatosplenomegaly $(p<0.001)$ and in anemia $(p=0.021)$, leucocytosis $(p=0.032)$, thrombocytopenia $(p=0.001)$, and elevated aspartate aminotransferase $(p=0.033)$. One of the main limitations of this study is using non-probability convenience sampling method since the choice of choosing antibiotic was left to physician's discretion. However, Doxycycline appears to be superior to Azithromycin in defervescing fever in Pediatric ST. Large scale randomized controlled trials (RCTs) are needed to confirm these preliminary findings.

\section{Compliance with Ethical Standards}

Conflict of Interest None.

Consent to Participate Written informed consent was obtained from the parents/legal guardians.

Consent to Publish Parents/legal guardians signed informed consent regarding publishing their child's data.

\section{References}

1. Rathi N, Kulkarni A, Yewale V. IAP guidelines on rickettsial diseases in children. Indian Pediatr. 2017;54:223-9.

2. El Sayed I, Liu Q, Wee I, Hine P. Antibiotics for treating scrub typhus. Cochrane Database Syst Rev. 2018;9:CD002150.

3. Wee I, Lo A, Rodrigo C. Drug treatment of scrub typhus: a systematic review and meta-analysis of controlled clinical trials. Trans Royal Soc Trop Med Hyg. 2017;111:336-44.

4. Biggs HM, Behravesh CB, Bradley KK, et al. Diagnosis and management of tickborne rickettsial diseases, CDC. MMWR Recomm Rep. 2016;65:1-44.

Publisher's Note Springer Nature remains neutral with regard to jurisdictional claims in published maps and institutional affiliations. 